\title{
Is the Carotid Artery a Window to the Left Ventricle?
}

\author{
Jon-Emile S Kenny
}

Keywords: Carotid ultrasound, Doppler ultrasound, Fluid responsiveness, Velocity time integral.

Indian Journal of Critical Care Medicine (2022): 10.5005/jp-journals-10071-24140

Sir,

I read with interest recent research by Chowhan et al. ${ }^{1}$ They ask whether the common carotid artery velocity time integral (cVTI) can be used to infer how the left ventricle responds to a passive leg raise (PLR) maneuver in critically ill patients categorized as either non-septic controls, septic or septic shock.

They observed that $\%$ change $(\% \Delta)$ in CVTI during PLR detected a $15 \%$ change in stroke volume (SV) in the non-septic, control group with an acceptable area under the receiver operator curve (AUROC) value of 0.74 . In the septic group, the $\% \Delta \mathrm{cVTI}$ had an excellent AUROC (i.e., 0.90) for detecting a 15\% change in SV, but in the septic shock group, the AUROC was only 0.69 . The optimal $\%$ $\Delta \mathrm{CVTI}$ thresholds for these three groups were approximately $11 \%$, $6 \%$, and $15 \%$, respectively. ${ }^{1}$

Yet, the authors also reported poor correlation between the CVTI and SV calculated by transthoracic echocardiography. This is slightly surprising given a recent study showing good correlation between $\% \Delta \mathrm{CVTI}$ and $\% \Delta$ LVOT VTI in critically ill patients receiving controlled, mechanical ventilation. ${ }^{2}$ Admittedly, the study of Pace et al. evaluated \% cVTI and \% aortic VTI variation during the respiratory cycle and not in response to the PLR. ${ }^{2}$ Nevertheless, in a human model of central hypovolemia and resuscitation comparing approximately 50,000 cardiac cycles, we observed a strong, linear correlation between $\% \Delta \mathrm{SV}$ measured by noninvasive pulse contour analysis and $\% \Delta$ CVTI measured by a wearable Doppler ultrasound. ${ }^{3}$ Why might the findings of Chowhan et al. be disparate?

The correlation that Chowhan et al. revealed in their Figure 2 is the change in (i.e., "delta") SV and cVTI. It appears that they regressed \% CVTI against \% SV, as they did for their AUROC analyses. However, there are zero data points above "delta SV 15" in the control group (their Fig. 2A) and only 1 data point above "delta SV $15^{\prime \prime}$ in the septic shock group (their Fig. 2C). Given that they report that 35\% (i.e., $7 / 20$ ) in the control group and 35\% (i.e., $7 / 20$ ) in the septic shock group were fluid responders-defined as an increase in SV of at least $15 \%$ - should not there be 7 data points in each of these groups above "delta SV 15?"

If, instead, Chowhan et al. regressed absolute change in SV (in milliliters) against absolute change in VTI (in centimeters), this relationship may not be a fair comparison and could explain discordance. Finally, the authors are absolutely correct to assert that time delay between measurements made at the LVOT and common carotid artery, ${ }^{4}$ inadequate beat sample size, ${ }^{5}$ human factors, ${ }^{4}$ and cerebral autoregulation all might, additionally, mediate discrepancy. Accordingly, cVTI may remain a useful, albeit
Department of Medicine, Health Sciences North Research Institute, Sudbury, Ontario, Canada

Corresponding Author: Jon-Emile S Kenny, Department of Medicine, Health Sciences North Research Institute, Sudbury, Ontario, Canada, e-mail: jon-emile@heart-lung.org

How to cite this article: Kenny JES. Is the Carotid Artery a Window to the Left Ventricle? Indian J Crit Care Med 2022;26(3):406.

Source of support: Nil

Conflict of interest: I am the Chief Medical Officer and co-founder of a start-up building a wearable Doppler ultrasound

transient, window to the left ventricle; this is most likely observed under ideal measurement conditions and when the unit of interest is relative change.

\section{ORCID}

Jon-Emile S Kenny 이 https://orcid.org/0000-0002-3654-1146

\section{References}

1. Chowhan G, Kundu R, Maitra S, Arora MK, Batra RK, Subramaniam R, et al. Efficacy of left ventricular outflow tract and carotid artery velocity time integral as predictors of fluid responsiveness in patients with sepsis and septic shock. Indian J Crit Care Med 2021;25(3):310-316. DOI: 10.5005/jp-journals-10071-23764.

2. Pace R, Lassola S, Miori S, Cammarota G, Barbariol F, Vetrugno L. Carotid vs aortic velocity time integral and peak velocity to predict fluid responsiveness in mechanically ventilated patients. A comparative study. Minerva Anestesiol 2021. DOI: 10.23736/S03759393.21.16035-3 [in press].

3. Kenny J-ÉS, Barjaktarevic I, Mackenzie DC, Elfarnawany M, Yang Z, Eibl AM, et al. Carotid Doppler ultrasonography correlates with stroke volume in a human model of hypovolaemia and resuscitation: analysis of 48570 cardiac cycles. $\mathrm{Br} J$ Anaesth 2021;127(2):e60-e63. DOI: 10.1016/j.bja.2021.05.007.

4. Kenny J-ÉS, Barjaktarevic I. Timing and measurement variability are critical when using carotid Doppler to infer hemodynamics. Ultrasound Med Biol 2020;46(12):3485-3486. DOI: 10.1016/ j.ultrasmedbio.2020.08.029.

5. Kenny J-ÉS, Barjaktarevic I, Mackenzie DC, Elfarnawany M, Math ZYB, Eibl AM, et al. Carotid Doppler measurement variability in functional hemodynamic monitoring: an analysis of 17,822 cardiac cycles. Crit Care Explor 2021;3(6):e0439. DOI: 10.1097/ CCE.0000000000000439.

(-) The Author(s). 2022 Open Access This article is distributed under the terms of the Creative Commons Attribution 4.0 International License (https://creativecommons. org/licenses/by-nc/4.0/), which permits unrestricted use, distribution, and non-commercial reproduction in any medium, provided you give appropriate credit to the original author(s) and the source, provide a link to the Creative Commons license, and indicate if changes were made. The Creative Commons Public Domain Dedication waiver (http://creativecommons.org/publicdomain/zero/1.0/) applies to the data made available in this article, unless otherwise stated. 